\title{
AN EVOLUTIONARY SCENARIO FOR SHORT PERIOD ( $\leq 10$ DAYS) MILLISECOND BINARY PULSARS
}

\author{
E. ERGMA ${ }^{1,2}$, M. J. SARNA ${ }^{1,3}$, J. ANTIPOVA ${ }^{1}$ \\ 1. Tartu University, Physics Department, Ülikooli 18, \\ EE2400, Tartu, Estonia \\ 2. NORDITA,Blegdamsvej 17, DK2100, Copenhagen 0, \\ Denmark \\ 3. N. Copernicus Astronomical Center, Polish Academy of \\ Science, ul. Bartycka 18, 00-716 Warsaw, Poland
}

\section{Discussion}

We present numerical calculations that simulate the evolution of a low mass $\left(1 \mathrm{M}_{\odot}\right.$ ) star transfering mass to a compact object (Muslimov \& Sarna 1993; Ergma \& Sarna 1996). Mass transfer starts when the secondary turns off the main sequence (having a small helium core). We have calculated 14 evolutionary sequences with the assumption of non-conservative or conservative evolution. We can conclude that near the bifurcation point the evolution is very sensitive to: (i) the assumption of conservative or non-conservative evolution, (ii) the structure of the mass losing star. Small changes in the initial period when the secondary fills its Roche lobe will lead to large changes in the final period and final mass of the remnant. Presently there are 40 known low-mass binary pulsars (LMBP). The evolutionary scenario for the wider systems $\left(10<P_{\text {orb }}(\mathrm{d})<1000\right)$ is connected with that of wide low-mass X-ray binaries (LMXB) in which the donor star will fill its Roche lobe after helium core formation. For such stars there is a simple relation between the mass of the degenerate helium core and the stellar radius (Refsdal \& Weigert 1971). The final orbital period is uniquely determined by the core mass of the final helium white dwarf (Joss, Rappaport \& Webbink 1987; Rappaport et al. 1995). As was pointed out by van den Heuvel \& Bitzaraki (1994) the picture described above is too simplified for systems with initial orbital periods ranging from approximately 0.5 to $2 \mathrm{~d}$. The recent discovery of the very short orbital period systems PSR J1012+5307 ( $P_{\text {orb }}=0.604 \mathrm{~d}$, $P_{\text {pul }}=5.26 \mathrm{~ms}, M=0.11 \mathrm{M}_{\odot}$; Nicastro et al. 1995) and PSR J0751+18 $\left(P_{\text {orb }}=0.263 \mathrm{~d}, P_{\mathrm{pul}}=3.48 \mathrm{~ms}, M=0.12 \mathrm{M}_{\odot} ;\right.$ Lundgren, Zepka \& Cordes 
1995) has raised the question of how to explain their past evolution. Our calculations support the model of Kluźniak, Czerny \& Ray (1992) in which the secondary star of PSR 1744-24A fills its Roche lobe when it turns off the main sequence. Due to the low accretion rate the system is in the 'propeller' phase which may explain its very erratic eclipsing behaviour. The orbital parameters of PSR J0751+18 may be explained if we assume nonconservative evolution starting from an initial period, $P_{\mathrm{i}}$, of $1.05 \mathrm{~d}$. After the accretion stops near $0.343 \mathrm{~d}$ the system will evolve due to gravitation wave radiation (GWR) and after $\sim 7.510^{9} \mathrm{yr}$ its orbital parameters will be as observed. We may explain the orbital parameters of PSR J1012+5307 if we assume again non-conservative evolution but this time starting with an initial period between $0.53 \ldots 0.63 \mathrm{~d}$. Since the orbital period is very large GWR is ineffective, and the system's orbital period will be as it was when the system detached.

Acknowledgements. EE would like to thank Drs. D. Lorimer, F. Camillo and S. Lundgren for providing the data for PSR J0751+18 and PSR J1012 +5307 .

\section{References}

Ergma, E., Sarna, M.J., 1996, MNRAS, in press

Joss, P.C., Rappaport, S.A., Webbink, R.F., 1987, Ap. J., 319, 180

Kluźniak, W., Czerny, M., Ray, A., 1992, in "X-ray Binaries and Recycled Pulsars" eds, E.P.J. van den Heuvel, S.A. Rappaport, p375

Lundgren, S.C., Zepka, A.F., Cordes, J.M., 1995, Ap. J., 453, 419

Muslimov, A.G., Sarna M.J., 1993, MNRAS, 262, 164

Nicastro, L., Lyne, A.G., Lorimer, D.R., et al., 1995, MNRAS, 273, L68

Rappaport, S.A., Podsiadlowski, P., Joss, P.C., et al., 1995, MNRAS, 273, 731

Refsdal, S., Weigert, A., 1971, A\&A, 6, 426

van den Heuvel, E.P.J., Bitzaraki, O., 1994, in "Evolutionary Relations in the Zoo of Interacting Binaries" eds, F. D" Antona, Mem. Soc. Astron. Ital., p237 